\title{
Nonlinear Circle-Criterion Observer Design for an Electrochemical Battery Model
}

\author{
Pierre Blondel $^{\circledR}$, Romain Postoyan, Stéphane Raël, Sébastien Benjamin, and Philippe Desprez
}

\begin{abstract}
Smart battery management systems require reliable information on the internal state of the battery, which are unavailable through direct measurements. Electrochemical models are relevant in this context as these describe the internal phenomena governing the battery dynamics. These models can thus be used to design observers, which provide estimates of the state variables online. In this paper, we consider a single particle electrochemical model of a lithium-ion battery given by a set of ordinary differential equations built from the spatial discretization of partial differential equations (PDEs) that locally describe the mass and charge transport of lithium. We then design a nonlinear observer, whose global exponential stability and robustness are guaranteed provided that a linear matrix inequality holds. The latter is shown to be verified for standard model parameters values. Simulation results based on plug-in hybrid electric vehicle tests on the original PDE model are presented, which illustrate the good performances of the observer in terms of speed of convergence and robustness.
\end{abstract}

Index Terms-Lithium batteries, Lyapunov methods, nonlinear observer, stability.

\section{INTRODUCTION}

$\mathbf{E}$ NERGY storage for embedded systems remains one of the technical challenges of the 21 st century. Introduced in the early 1990s, lithium-ion (Li-ion) batteries are widely used and will play a key role in energetic transition. Compared with other electrochemical devices, Li-ion batteries exhibit a high-weight power density, a high-volume power density, a low self-discharge current, and do not suffer from the memory effect. On the other hand, this technology requires

Manuscript received June 21, 2017; revised October 11, 2017; accepted November 15, 2017. Manuscript received in final form December 10, 2017. This work was supported by the Association Nationale de la Recherche et de la Technologie. Recommended by Associate Editor A. Pavlov. (Corresponding author: Pierre Blondel.)

P. Blondel is with the Centre de Recherche en Automatique de Nancy (CRAN), UMR 7039, Université de Lorraine, 54506 Vandœuvre-lès-Nancy, France, with the Centre national de la recherche scientifique, CRAN, UMR 7039, 54506 Vandœuvre-lès-Nancy, France, also with the Groupe de Recherche en Électrotechnique et Électronique de Nancy, Université de Lorraine, 54506 Vandoeuvre-lès-Nancy, France, and also with the Saft Corporate Research Center, 33300 Bordeaux, France (e-mail: pierre.blondel@univlorraine.fr).

R. Postoyan is with the Centre de Recherche en Automatique de Nancy (CRAN), UMR 7039, Université de Lorraine, 54506 Vandœuvre-lès-Nancy, France, and also with the Centre national de la recherche scientifique, CRAN, UMR 7039, 54506 Vandœuvre-lès-Nancy, France (e-mail: romain.postoyan@univ-lorraine.fr).

S. Raël is with the Groupe de Recherche en Électrotechnique et Électronique de Nancy, Université de Lorraine, 54506 Vandoeuvre-lès-Nancy, France (e-mail: stephane.rael@univ-lorraine.fr).

S. Benjamin and P. Desprez are with the Saft Corporate Research Center, 33300 Bordeaux, France (e-mail: sebastien.benjamin@saftbatteries.com; philippe.desprez@saftbatteries.com).

Color versions of one or more of the figures in this paper are available online at http://ieeexplore.ieee.org.

Digital Object Identifier 10.1109/TCST.2017.2782787 a careful management in terms of safety. This is the purpose of the so-called battery management systems (BMS), which also provides to the user useful information on the state of the battery, such as the state of charge (SOC) and the state of health $(\mathrm{SOH})$ to mention a few.

A way to improve existing BMS is to exploit models, which describe the internal dynamics of the battery. Two families of models can be distinguished. The first category relies on equivalent circuit models (ECMs), which are simple electrical analogies of the battery dynamics that essentially consist of a few coupled resistors and capacitors in series and parallel. The design of these ECM-based techniques is usually easy but the models are simplistic and nontrivial parameterization is needed, which can either be data-driven [1] or done by a bank of Kalman filters as in [2] or [3].

The second category of models are the electrochemical ones, which describe the lithium concentrations in different locations. These models offer a more faithful description of the battery dynamics but are usually more difficult to exploit for the BMS. In this paper, we consider such models, as we are convinced that electrochemical models are a promising step toward the developments of the advanced BMS. Doyle et al. [4] propose a full electrochemical model known as pseudo-2-D model, which locally describes the electrochemical phenomena through coupled partial differential equations (PDEs). Improvements of this work have been done in [5] and [6] by extending the domain of validity and the generality of these models. Zou et al. [7] develop a comprehensive PDE battery model, which captures the coupled electrochemical, thermal, and capacity-fade dynamics, and they present a general methodology to obtain reduced models, inspired by the singular perturbation theory, to adapt the complexity of the PDE model to any kind of Li-ion battery technology or any specific applications.

While PDE models provide an accurate description of the battery dynamics, solving these models remains computationally intensive, which may make their implementation on embedded devices difficult. A specific reduction of these models, known as single particle models (SPMs), where each electrode is approximated as a spherical particle, is often used [8], [9]. This type of models is less accurate but computationally lighter than the previous ones and has been used for the development of various estimation techniques, see the PDE estimator in [9] and [10], the extended Kalman filter in [8], the unscented Kalman filter in [11], or the deterministic finitedimensional observer [12].

In this paper, we aim at designing an observer for a finitedimensional SPM like in [8] and [12]. To work with such 
models may be essential in practice to make the implementation of the observer on embedded devices possible. Inspired by [13], the SPM-like model we develop has the current applied to the battery as the input and the battery voltage, which is a nonlinear function with the states and the input as arguments, as output. Its state dynamics are described by a set of affine ordinary differential equations (ODEs) obtained by the spatial discretization of the PDE governing the mass diffusion of the lithium in its particle as in [8]. The statespace representation takes the form of an affine dynamical system with a nonlinear output map. This model differs from the SPM model in [12], for instance, where a similar problem is addressed. Indeed, in [12], the considered model only describes the diffusion phenomenon in the negative electrode. The surface concentration of the positive electrode, which is needed to compute the output voltage, is obtained by a transfer function based on the mass conservation of lithium. In our model, both the electrodes are described, we can therefore access to the lithium concentration in these and compute the output voltage directly. Another main difference in [8] and [12] is the geometric exactness of the spatial discretization we choose, which guarantees the mass conservation of the lithium.

We then design an observer for the obtained model. The key difficulty is that the output map is nonlinear. It appears that none of the observer designs for systems with nonlinear output maps we are aware of are applicable to our system, either because the model is not of the right form [14], or because the required assumptions are not satisfied [12], [15]. We, therefore, propose a new observer, which is inspired by the designs in [16] and [17] where circle-criterion arguments are used. The key idea is to write the state-dependent term of the output map as the sum of an affine function and nonlinear functions satisfying sector conditions. This formulation is possible in our case as the output map is composed of the open circuit voltage (OCV) versus state maps and some current-dependent terms. We then design the observer, provided that a linear matrix inequality (LMI) holds, which is shown to be the case for standard model parameter values. We ensure that the origin of the state estimation error system is uniformly globally exponentially stable (UGES) in the absence of exogenous inputs and noise, otherwise we guarantee a $\mathcal{L}_{2}$-stability property. Contrary to [8] and [11], global stability of the observer is established, which is also crucial for critical applications. The design method we present is first derived by assuming the battery temperature constant. We then explain how to extend these results to the case where the temperature varies within a given (potentially large) interval by using a polytopic approach. We have finally implemented the observer on the original PDE model. Good estimation performances are obtained, even for high currents and noisy measurements.

In our preliminary work in [18], we considered the same SPM but we designed the observer very differently, using a polytopic approach instead of invoking circle-criterion arguments. As a result, the LMI condition presented in this paper is different and has a much smaller dimension. We also think that the design we present in this paper is a contribution in its own right as it extends the results in [17] and [19] to the case where the output map is nonlinear. In addition, we consider the impact of temperature, which is not the case in [18].

Notations: Let $\mathbb{R}:=(-\infty, \infty), \mathbb{R}_{\geq 0}:=[0, \infty)$, and $\mathbb{R}_{>0}:=(0, \infty)$. We use $I_{n}$ to denote the identity matrix of size $n$. For $A \in \mathbb{R}^{N \times M}, A^{T}$ stands for the transpose of $A$. For $x \in \mathbb{R}^{N}$ and $y \in \mathbb{R}^{M},(x, y)$ denotes $\left(x^{T}, y^{T}\right)^{T}$. For any symmetric matrix $A \in \mathbb{R}^{N \times N}, \lambda_{\max }(A)$ stands for the biggest eigenvalue of $A$. The symbol $*$ in a matrix is for the symmetric term, i.e. $\left[\begin{array}{cc}A & B \\ * & C\end{array}\right]=\left[\begin{array}{cc}A & B \\ B^{T} & C\end{array}\right]$. For $x \in \mathbb{R}^{N},|x|$ stands for the Euclidean norm of $x$. Let $f: \mathbb{R}^{+} \rightarrow \mathbb{R}^{n},\|f\|_{2,[0, t)}$ denote the $\mathcal{L}_{2}$ norm of $f$ on the interval $[0, t)$, where $t \in[0, \infty)$, i.e., $\|f\|_{2,[0, t)}=\left(\int_{0}^{t}|f(s)|^{2} d s\right)^{1 / 2}$, when it is well-defined. We write that $f \in \mathcal{L}_{2}$, when $\|f\|_{2,[0, \infty)}<\infty$.

\section{Electrochemical Model}

The electrochemical model we consider describes the solid diffusion of the lithium that occurs in the solid phase of the electrodes. It is derived from a PDE-based model given in [13] and takes the form of a finite-dimensional nonlinear statespace representation.

In this section, we describe the system, then we derive the model of the solid diffusion of lithium in the electrodes, and finally, we give the output of the model. The parameters used in this section are defined in Table I in Appendix B.

\section{A. Description}

A Li-ion battery cell is composed of the negative electrode, the positive electrode, the separator, and the electrolyte. The electrodes are separated by the well-named separator and those three components are immersed in the electrolyte. The electrolyte is an ionic solution that can exchange lithium with the electrodes and provides electrical insulation. Each electrode has a certain potential due to the electrochemical couple formed by its material and the lithium dissolved in the electrolyte. Electrons are attracted by this difference of potential but cannot be exchanged from one electrode to the other within the battery unlike lithium ions. An electron that belongs to an electrode can go through an external electrical circuit to reach the other electrode if this circuit exists. Then, lithium is removed from its source electrode and another is inserted in its electrode of destination. This conserves the charges' equilibrium in the electrodes and in the electrolyte at any time. From a macroscopic point of view, this flow of electrons between the electrodes through an external circuit corresponds to the current.

\section{B. Assumptions}

To simplify the electrochemical model, we assume that the behavior of a single particle of the average size of the particles in the electrode represents the behavior of the whole electrode as in [8]-[13] and [18], which corresponds to the next assumption.

Standing Assumption 1 (SA1): Each electrode in the model is composed of a single particle, which is a sphere of the average size of the particles that compose the actual electrode. 


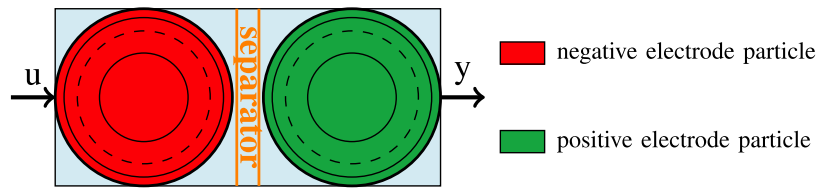

Fig. 1. SPM model schematic.

SA1 neglects the thickness of the electrode, allowing a significant reduction in the order of the model and leads to a friendly state-space form as we will show. Again, we will see in Section III that although the observer will be designed on a simplified model, good performances are obtained in simulations of a PDE model, which does not rely on SA1.

Remark 1: In [12], only one particle is considered and the surface concentration at the positive electrode, needed for output computation, is deduced from the surface concentration of the negative electrode thanks to a transfer function while we estimate the lithium concentration in both the electrodes like in [8]. The proposed model, therefore, describes in more detail the concentration of lithium in the positive electrode. $\square$

We also make the next assumption on the electrolyte.

Standing Assumption 2 (SA2): The electrolyte is neglected.

Model simulations with a standard set of parameters used in this paper show that the contribution of the electrolyte to the dynamics of the model and to the output voltage is negligible for reasonable currents. SA2 simplifies the model and has a low impact on its accuracy. Fig. 1 shows the SPM model under SA2 and SA1.

We also assume that the model parameters are known and leave the estimation of some of these, like diffusions coefficients, for future work.

Standing Assumption 3 (SA3): All the parameters of the model are known.

\section{Solid Diffusion in a Particle}

In view of SA1, the main physical phenomenon is the lithium diffusion in the electrodes. Lithium diffusions in the negative and positive electrodes are ruled by the same physical law, only the parameterization changes from one electrode to the other.

According to SA1, the negative and positive particles are spheres. We introduce the subscript $s \in\{$ neg, pos\} for the sake of convenience. The concentration of solid lithium with respect to $r$, the radial coordinate, is driven by the solid diffusion equation, which is described by the PDE

$$
\frac{\partial c_{s}(r, t)}{\partial t}=\frac{1}{r^{2}} \frac{\partial}{\partial r}\left[D_{s} r^{2} \frac{\partial c_{s}(r, t)}{\partial r}\right],
$$

where $c_{s}$ is the lithium concentration in the solid phase, along with two boundaries conditions,

$$
\left.\frac{\partial c_{s}(r)}{\partial r}\right|_{r=0}=\left.0 \quad \frac{\partial c_{s}(r)}{\partial r}\right|_{r=R_{s}}=K_{I}^{s} I
$$

with $I$ the current, $a_{s}:=\left(3 \varepsilon_{s} / R_{s}\right)$, and under active sign convention, $K_{I}^{\text {neg }}=-1 /\left(D_{\text {neg }} a_{\text {neg }} F \mathcal{A}_{\text {cell }} d_{\text {neg }}\right)$ and $K_{I}^{\text {pos }}=$ $1 /\left(D_{\text {pos }} a_{\text {pos }} F \mathcal{A}_{\text {cell }} d_{\text {pos }}\right)$. All the subscripts, superscripts, and indexes are given in Table II in Appendix B. The boundary condition at $r=0$ means that there are no lithium flux in the center of the sphere for geometrical reasons. The boundary condition in $r=R_{s}$ means that the flux through the surface of the sphere is proportional to the battery current.

We spatially discretize (1) and (2) to obtain a set of ODEs. We consider $N_{s}$ samples where the lithium concentration is considered constant in each particle. The size of these samples is voluntarily not given because any sampling rule can be chosen to discretize the particles. We intend to accurately estimate the surface concentrations in this paper, consequently samples with identical volumes are chosen in Section IV to generate the numerical values of the electrochemical model. This sampling rule tends to tighten the thickness of the samples near the surface of the particles. Samples with identical radius are also relevant for other purposes [12].

Let $S_{n}$ and $V_{n}$ be the external surface and the volume of the sample $n \in\left\{1, \ldots, N_{s}\right\}$, respectively. We spatially discretize (1) and (2) using right-sided Newton's difference quotient, for $n \in\left\{2, \ldots, N_{s}-1\right\}$,

$$
\begin{aligned}
\frac{d c_{n}}{d t} & \\
= & \frac{S_{n-1}}{r_{n}-r_{n-1}} \frac{D_{s}}{V_{n}} c_{n-1}-\left(\frac{S_{n-1}}{r_{n}-r_{n-1}}+\frac{S_{n}}{r_{n+1}-r_{n}}\right) \frac{D_{s}}{V_{n}} c_{n} \\
& +\frac{S_{n}}{r_{n+1}-r_{n}} \frac{D_{s}}{V_{n}} c_{n+1},
\end{aligned}
$$

for $n=1$

$$
\frac{d c_{1}}{d t}=\frac{S_{1}}{r_{2}-r_{1}} \frac{D_{s}}{V_{1}}\left(-c_{1}+c_{2}\right),
$$

and for $n=N_{s}$

$$
\frac{d c_{N_{s}}}{d t}=\frac{S_{N_{s}-1}}{r_{N_{s}}-r_{N_{s}-1}} \frac{D_{s}}{V_{N_{s}}}\left(c_{N_{s}-1}-c_{N_{s}}\right)+\bar{K}_{I}^{s} I,
$$

with $\bar{K}_{I}^{\text {neg }}=-\frac{S_{\text {neg }}}{V_{\text {neg }} a_{\text {neg }} F \mathcal{A}_{\text {cell }} d_{\text {neg }}}$ and $\bar{K}_{I}^{\text {pos }}=\frac{S_{\text {pos }}}{V_{\text {pos }} a_{\text {pos }} F \mathcal{A}_{\text {cell }} d_{\text {pos }}}$.

Remark 2: In [8] and [12], geometrical approximations in the volumes and surfaces of the samples lead to some modeling uncertainties contrary to what our model considers. Indeed, to ensure the geometric accuracy and mass conservation of the lithium in our model, the volumes $V_{n}$ and the surfaces $S_{n}$ are analytically obtained by integration along the $r$ coordinate. $\square$

The battery does not acquire or leak lithium materials over short time horizons. A mass conservation of the lithium in solid-phase equation can therefore be written. Let $N_{\text {neg }}$ and $N_{\text {pos }}$ be the number of samples of the negative and positive electrodes radially discretized, respectively. The quantity of lithium in the solid phase is defined as

$$
Q:=\alpha_{\text {neg }} \sum_{i=1}^{N_{\text {neg }}} c_{i}^{\text {neg }} V_{i}^{\text {neg }}+\alpha_{\text {pos }} \sum_{i=1}^{N_{\text {pos }}} c_{i}^{\text {pos }} V_{i}^{\text {pos }},
$$

with $\quad \alpha_{\text {neg }} \quad:=(F / 3600)\left(\varepsilon_{s, \text { neg }} \mathcal{A}_{\text {cell }} d_{\text {neg }} / V_{\text {total }}^{\text {neg }}\right), \quad$ and $\alpha_{\text {pos }}:=(F / 3600) \quad\left(\varepsilon_{s, \text { pos }} \mathcal{A}_{\text {cell }} d_{\text {pos }} / V_{\text {total }}^{\text {pos }}\right), \quad$ with $V_{\text {total }}^{s}=\frac{4}{3} \pi R_{s}^{3}$.). The term $Q$ is expressed in Ah, which explains the scaling term $(F / 3600)$ in $\alpha_{\text {neg }}$ and $\alpha_{\text {pos }}$.

To reduce the order of the model, we express the concentration of lithium at the center of the negative electrode $c_{1}^{\text {neg }}$ 

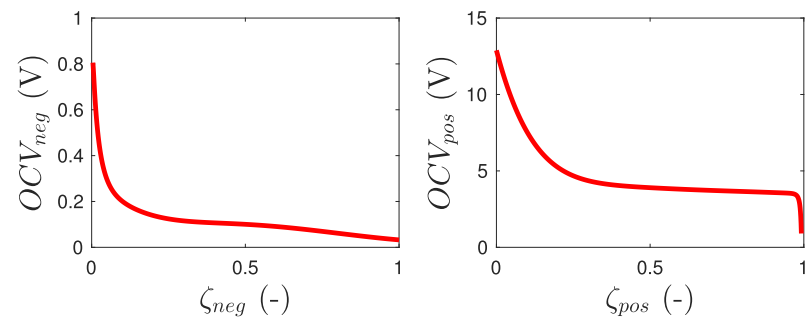

Fig. 2. OCV curves extracted from an empirical formula given by [5].

as a linear combination of all the other sampled concentration in the solid phase

$$
c_{1}^{\text {neg }}=\bar{K}+\sum_{i=2}^{N_{\text {neg }}} \beta_{i}^{\text {neg }} c_{i}^{\text {neg }}+\sum_{i=1}^{N_{\text {pos }}} \beta_{i}^{\text {pos }} c_{i}^{\text {pos }},
$$

with $\bar{K}:=\left(Q / \alpha_{\text {neg }} V_{1}^{\text {neg }}\right), \beta_{i}^{\text {neg }}:=-\left(V_{i}^{\text {neg }} / V_{1}^{\text {neg }}\right)$ and $\beta_{i}^{\text {pos }}:=$ $-\left(\alpha_{\text {pos }} V_{i}^{\text {pos }} / \alpha_{\text {neg }} V_{1}^{\text {neg }}\right)$. This simple reduction appears to be very useful to ensure the satisfaction of the conditions for the stability of the observer in Section III.

Let $x:=\left(c_{2}^{\text {neg }}, \ldots, c_{N_{\text {neg }}^{\text {neg }}}^{\text {nos }}, c_{1}^{\text {pos }}, \ldots, c_{N_{\text {pos }}}^{\text {pos }}\right) \in \mathbb{R}^{N}$ with $N:=$ $N_{\text {neg }}-1+N_{\text {pos }}$ be the vector of lithium concentration in each sample of both the electrodes and $u \in \mathbb{R}$ be the current $I$. From (3), (5), and (7), we obtain

$$
\dot{x}=A x+B u+K,
$$

where $A \in \mathbb{R}^{N \times N}, B \in \mathbb{R}^{N}$, and $K \in \mathbb{R}^{N}$ are constant matrices whose expressions follow from (3), (5), and (7); recall that all parameters are known according to SA3.

\section{Output Voltage}

The output voltage is the output of our model. The main components of the output voltage are the potential differences between the electrodes and the electrolyte without current, called OCV, which vary with the lithium concentration at the surface of the electrodes.

In view of SA1, the OCV depend on the insertion rate of the lithium defined for the negative and positive electrodes as

$$
\zeta_{\text {neg }}:=c_{\text {neg }}^{\text {surf }} / c_{\text {neg }}^{\max }
$$

and

$$
\zeta_{\mathrm{pos}}:=c_{\mathrm{pos}}^{\mathrm{surf}} / c_{\mathrm{pos}}^{\max }
$$

Fig. 2 shows standard OCV curves for Li-ion batteries. Let $H_{\text {neg }}:=\left(\begin{array}{llllll}0 & \ldots & 0 & 1 / c_{\text {neg }}^{\max } 0 & \ldots & 0\end{array}\right) \in \mathbb{R}^{1 \times n_{x}}$, where the nonzero component is at the $N_{\text {neg }}-1$ position and $H_{\text {pos }}:=$ $\left(0 \ldots 01 / c_{\text {pos }}^{\max }\right) \in \mathbb{R}^{1 \times n_{x}}$. Matrices $H_{\text {neg }}$ and $H_{\text {pos }}$ select and scale the surface concentration to provide the OCV functions the corresponding insertions rates as argument, hence, we have $H_{\text {neg }} x=\zeta_{\text {neg }}$ and $H_{\text {pos }} x=\zeta_{\text {pos }}$, respectively. The output voltage, $y \in \mathbb{R}$, is obtained from those maps knowing the surface lithium concentration of each particles and the current, leading to

$$
y:=\mathrm{OCV}_{\text {pos }}\left(H_{\mathrm{pos}} x\right)-\mathrm{OCV}_{\text {neg }}\left(H_{\mathrm{neg}} x\right)+g(u),
$$

where $g(u)$ is the current-dependent component of the output, whose expression is provided in Appendix A.

\section{E. Temperature}

Some parameters arising in (8) and (9) depend on the temperature $\theta$, like the diffusion coefficients $D_{s}, s \in\{$ neg, pos $\}$. In practice, the temperature is usually measured, we therefore assume that we know it. In Section III, we will first derive a nonlinear observer in the case where $\theta$ is constant over the time. We will then explain how to extend these results to the case where $\theta$ varies with time.

\section{OBSERVER DESIGN AND ANALYSIS}

\section{A. Constant Temperature}

When the temperature $\theta$ is constant, system (8) and (9) is of the form

$$
\left\{\begin{array}{l}
\dot{x}=A x+B u+K+E w \\
y=h(x)+g(u)+z,
\end{array}\right.
$$

where $h: \mathbb{R}^{n_{x}} \rightarrow \mathbb{R}$ is continuous, $w \in \mathbb{R}^{n_{w}}$ is a vector of exogenous perturbations, which may model errors due to parameter uncertainties, and $z \in \mathbb{R}^{n_{z}}$ models the measurement noise.

Remark 3: The matrix $A$ is not Hurwitz in (10). Hence, the observer presented in [12] is not applicable to this model as conditions (23) and (37) are not satisfied.

We propose the observer

$$
\left\{\begin{array}{l}
\dot{\hat{x}}=A \hat{x}+B u+K+L(y-\hat{y}) \\
\hat{y}=h(\hat{x})+g(u)
\end{array}\right.
$$

where $\hat{x} \in \mathbb{R}^{n_{x}}$ is the state estimate and $L \in \mathbb{R}^{n_{x} \times 1}$ is the observation matrix gain to be designed; recall that $n_{x}$ is the dimension of the vector $x$ ( $n_{x}=N$ in Section II).

Let $e:=x-\hat{x}$ denote the estimation error. In view of (10) and (11)

$$
\dot{e}=A e+E w-L(h(x)-h(\hat{x}))-L z .
$$

We make Assumption 1 on the output map $h$.

Assumption 1: There exist $C \in \mathbb{R}^{1 \times n_{x}}$ and $\delta_{\text {neg }}^{\max }, \delta_{\text {pos }}^{\max } \in$ $\mathbb{R}_{\geq 0} \cup\{\infty\}$ such that for any $x, x^{\prime} \in \mathbb{R}^{n_{x}}$

$$
\begin{aligned}
h(x)-h\left(x^{\prime}\right)=C\left(x-x^{\prime}\right)+ & \delta_{\mathrm{neg}}\left(x, x^{\prime}\right) H_{\mathrm{neg}}\left(x-x^{\prime}\right) \\
& +\delta_{\mathrm{pos}}\left(x, x^{\prime}\right) H_{\mathrm{pos}}\left(x-x^{\prime}\right),
\end{aligned}
$$

where $\delta_{\text {neg }}\left(x, x^{\prime}\right) \in\left[0, \delta_{\text {neg }}^{\max }\right]$ and $\delta_{\text {pos }}\left(x, x^{\prime}\right) \in\left[0, \delta_{\text {pos }}^{\max }\right]$.

Assumption 1 plays a fundamental role in the design of the gain $L$ in (11). It essentially means that the output can be written as a sum of an affine term $C x+C^{0}$ for some $C^{0} \in \mathbb{R}$, and a monotone nonlinearity. As a result, the difference between any two values of $h$ can be written as the sum of a linear term and nonlinearities subject to sector conditions. We allow $\delta_{s}^{\max }$ to be equal to $\infty$ with $s \in\{$ neg, pos $\}$ in which case we use the convention $\left(1 / \delta_{s}^{\max }\right)=0$ in the following. We claim that Assumption 1 is always satisfied for any battery model with the output map given by (9). We can proceed as follows to verify it. We write $O C V_{\text {pos }}\left(\zeta_{\text {pos }}\right)=C_{\text {pos }} \zeta_{\text {pos }}+C_{\text {pos }}^{0}+v_{\text {pos }}\left(\zeta_{\text {pos }}\right)$ for any $\zeta_{\text {pos }} \in[0,1],{ }^{1}$ where

$$
C_{\mathrm{pos}}=\min _{\zeta \mathrm{pos} \in[0,1]}\left(\frac{d O C V_{\mathrm{pos}}\left(\zeta_{\mathrm{pos}}\right)}{d \zeta_{\mathrm{pos}}}\right),
$$

${ }^{1} \mathrm{OCV}$ curves are the function of the insertions rates, which are the surface concentration normalized with respect to the maximal admissible lithium concentration (see Fig. 2). That is the reason why $\zeta_{s}$ takes values in $[0,1]$. 
and

$$
C_{\mathrm{pos}}^{0}=-\min _{\zeta \mathrm{pos} \in[0,1]}\left(O C V_{\mathrm{pos}}\left(\zeta_{\mathrm{pos}}\right)-C_{\mathrm{pos}} \zeta_{\mathrm{pos}}\right)
$$

This way $\left(d v_{\text {pos }}\left(\zeta_{\text {pos }}\right) / d \zeta_{\text {pos }}\right) \geq 0$ for any $\zeta_{\text {pos }} \in[0,1]$. We used a similar procedure ${ }^{2}$ for $O C V_{\text {neg }}$ in (9). Consequently, $h(x)=C x+C^{0}+v_{\text {neg }}\left(H_{\text {neg }} x\right)+v_{\text {pos }}\left(H_{\text {pos }} x\right)$. Assumption 1 then follows by the application of the mean value theorem.

Using Assumption 1, (12) becomes

$$
\dot{e}=\left(A-L\left(C+\delta_{\text {neg }} H_{\text {neg }}+\delta_{\text {pos }} H_{\text {pos }}\right)\right) e+E w-L z,
$$

where we omit the arguments of $\delta_{\text {neg }}$ and $\delta_{\text {pos }}$ for the sake of convenience.

Theorem 1 ensures stability properties for system (10), provided we can find matrix $L$ verifying a given LMI.

Theorem 1: Suppose Assumption 1 holds and consider system (14). If there exist $\varepsilon, m_{\mathrm{neg}}, m_{\mathrm{pos}}, \mu_{w}, \mu_{z} \in \mathbb{R}_{>0}$, and $P \in \mathbb{R}^{n_{x} \times n_{x}}$ symmetric and positive-definite such that

$$
\left(\begin{array}{ccccc}
\mathcal{G}+\varepsilon I_{n_{x}} & \mathcal{K}_{\mathrm{neg}} & \mathcal{K}_{\mathrm{pos}} & P E & -P L \\
* & -\frac{2 m_{\mathrm{neg}}}{\delta_{\mathrm{neg}}^{\max }} & 0 & 0 & 0 \\
* & * & -\frac{2 m_{\mathrm{pos}}}{\delta_{\mathrm{pos}}^{\max }} & 0 & 0 \\
* & * & * & -\mu_{w} I_{n_{w}} & 0 \\
* & * & * & * & -\mu_{z} I_{n_{z}}
\end{array}\right)<0,
$$

where $\mathcal{G}:=(A-L C)^{T} P+P(A-L C), \mathcal{K}_{\text {neg }}:=-P L+$ $m_{\text {neg }} H_{\text {neg }}^{T}$, and $\mathcal{K}_{\text {pos }}:=-P L+m_{\text {pos }} H_{\text {pos }}^{T}$, then system (14) is $\mathcal{L}_{2}$-stable from $w$ and $z$ to $e$ with the gain less than or equal to $\left(\mu_{w} / \varepsilon\right)^{1 / 2}$ and $\left(\mu_{z} / \varepsilon\right)^{1 / 2}$, respectively, i.e., there exists $c \geq 0$ such that for any initial condition $e_{0} \in \mathbb{R}^{n_{x}}$, any $w, z \in \mathcal{L}_{2}$, the corresponding solution $e$ to (14) verifies for any $t \geq 0$

$$
\|e\|_{2[0, t)} \leq c\left|e_{0}\right|+\sqrt{\frac{\mu_{w}}{\varepsilon}}\|w\|_{2,[0, t)}+\sqrt{\frac{\mu_{z}}{\varepsilon}}\|z\|_{2,[0, t)} .
$$

Furthermore, when $w=0$ and $z=0, e=0$ is UGES, i.e., there exist $\gamma_{1}, \gamma_{2} \in \mathbb{R}_{>0}$, such that for any $e_{0} \in \mathbb{R}^{n_{x}}$, the corresponding solution $e$ to (14) satisfies $|e(t)| \leq \gamma_{1}\left|e_{0}\right| e^{-\gamma_{2} t}$ for any $t \geq 0$.

Proof: Let $e \in \mathbb{R}^{n_{x}}, w \in \mathbb{R}^{n_{w}}, z \in \mathbb{R}^{n_{z}}$, and $V(e):=$ $e^{T} P e$, where $P$ comes from Theorem 1 . Let $\mathcal{Z}:=A-L(C+$ $\left.\delta_{\text {neg }} H_{\text {neg }}+\delta_{\text {pos }} H_{\text {pos }}\right)$, in view of (14)

$$
\begin{aligned}
& \langle\nabla V(e), \mathcal{Z} e+E w-L z\rangle \\
& =\chi^{T}\left(\begin{array}{ccccc}
\mathcal{G} & -P L & -P L & P E & -P L \\
* & 0 & 0 & 0 & 0 \\
* & * & 0 & 0 & 0 \\
* & * & * & 0 & 0 \\
* & * & * & * & 0
\end{array}\right) \chi,
\end{aligned}
$$

\footnotetext{
${ }^{2}$ In this case, $C_{\text {neg }}=\max _{\zeta \text { neg } \in[0,1]}\left(d O C V_{\text {neg }}(\zeta\right.$ neg $) / d \zeta$ neg $)$ and $C_{\text {neg }}^{0}=$ $\min _{\zeta \text { neg }} \in[0,1]\left(O C V_{\text {neg }}\left(\zeta_{\text {neg }}\right)-C_{\text {neg }} \zeta_{\text {neg }}\right)$.
}

where $\chi:=\left(e, \delta_{\text {neg }} H_{\text {neg }} e, \delta_{\text {pos }} H_{\text {pos }} e, w, z\right)$. We derive from (15)

$$
\begin{aligned}
&\langle\nabla V(e), \mathcal{Z} e+E w-L z\rangle \\
& \leq \chi^{T}\left(\begin{array}{ccccc}
-\varepsilon I_{n_{x}} & -m_{\mathrm{neg}} H_{\mathrm{neg}}^{T} & -m_{\mathrm{pos}} H_{\mathrm{pos}}^{T} & 0 & 0 \\
* & \frac{2 m_{\mathrm{neg}}}{\delta_{\mathrm{neg}}^{\max }} & 0 & 0 & 0 \\
* & * & \frac{2 m_{\mathrm{pos}}}{\delta_{\mathrm{pos}}^{\max }} & 0 & 0 \\
* & * & * & \mu_{w} I_{n_{w}} & 0 \\
* & * & * & * & \mu_{z} I_{n_{z}}
\end{array}\right) \chi \\
&=-\varepsilon|e|^{2}+\mu_{w}|w|^{2}+\mu_{z}|z|^{2}-2 m_{\mathrm{neg}}\left(H_{\mathrm{neg}} e\right)^{2} \delta_{\mathrm{neg}} \\
& \quad \times\left(1-\frac{\delta_{\mathrm{neg}}}{\delta_{\mathrm{neg}}^{\max }}\right)-2 m_{\mathrm{pos}}\left(H_{\mathrm{pos}} e\right)^{2} \delta_{\mathrm{pos}}\left(1-\frac{\delta_{\mathrm{pos}}}{\delta_{\mathrm{pos}}^{\max }}\right) .
\end{aligned}
$$

According to Assumption $1,0 \leq \delta_{\text {neg }} \leq \delta_{\text {neg }}^{\max }$ and $0 \leq \delta_{\text {pos }} \leq$ $\delta_{\text {pos }}^{\max }$. Furthermore, $m_{\text {neg }}, m_{\text {pos }} \in \mathbb{R}_{>0}$, hence

$$
\langle\nabla V(e), \mathcal{Z} e+E w-L z\rangle \leq-\varepsilon|e|^{2}+\mu_{w}|w|^{2}+\mu_{z}|z|^{2} .
$$

Note that solutions to (14) are defined for any initial condition, any positive time, and any piecewise continuous inputs $w$ and $z$ in view of Assumption 1, according to [20, Th. 3.2]. In view of (19), for any $e_{0} \in \mathbb{R}^{n_{x}}, w, z \in \mathcal{L}_{2}$, the corresponding solution of $e$ to (14) verifies for any $t \geq 0$

$$
\dot{V}(e(t)) \leq-\varepsilon|e(t)|^{2}+\mu_{w}|w(t)|^{2}+\mu_{z}|z(t)|^{2},
$$

from which we deduce that

$\|e\|_{2,[0, t]} \leq \sqrt{\frac{\mu_{w}}{\varepsilon}}\|w\|_{2,[0, t)}+\sqrt{\frac{\mu_{z}}{\varepsilon}}\|z\|_{2,[0, t)}+\sqrt{\lambda_{\max }(P)}\left|e_{0}\right|$,

which corresponds to (16) with $c=\left(\lambda_{\max }(P)\right)^{1 / 2}$. When $w=0$ and $z=0$, we immediately deduce that $e=0$ is UGES for system (14) from (19) and the definition of $V$.

Theorem 1 means that, if we can design $L$ to satisfy (15), the state estimates provided by (11) uniformly, globally, and exponentially converge to the solutions to (10) when $w=0$ and $z=0$. When $(w, z) \neq 0$, the state estimation error system (14) is $\mathcal{L}_{2}$-stable from $w$ and $z$ to $e$ with gains less than or equal to $\left(\mu_{w} / \varepsilon\right)^{1 / 2}$ and $\left(\mu_{z} / \varepsilon\right)^{1 / 2}$, respectively. In other words, the observer is robust to $w$ and $z$ and these gains quantify this property. Condition (15) is a matrix inequality, which becomes linear after a standard change in variables, namely $W=P L$, so that standard LMI solvers can then be used to solve it. We can then use (15) to minimize the $\mathcal{L}_{2}$-gains associated with the model disturbance $w$ and the noise $z$, i.e., $\left(\mu_{w} / \varepsilon\right)^{1 / 2}$ and $\left(\mu_{z} / \varepsilon\right)^{1 / 2}$. In that case, we fix $\varepsilon$, and minimize the weighted sum of $\mu_{w}$ and $\mu_{z}$ for instance.

\section{B. Varying Temperature}

We now consider the scenario where the battery temperature $\theta$ varies with time. We recall that we assume that we measure $\theta$ (see Section II-E). We make Assumption 2 on $\theta$.

Assumption 2: There exists $\theta_{\min }, \theta_{\max }$ such that $\theta(t) \in$ $\left[\theta_{\min }, \theta_{\max }\right]$ for any $t \geq 0$. 
Assumption 2 is often met in practice as the battery constructors define the range of operating temperatures of their products and provide mechanisms to respect safety thresholds.

As a result, we write the corresponding system (8) and (9) as

$$
\left\{\begin{array}{l}
\dot{x}=A(\theta) x+B(\theta) u+K(\theta)+E w \\
y=h(x, \theta)+g(u, \theta)+z .
\end{array}\right.
$$

The observer used to estimate the state of system (22) is

$$
\left\{\begin{array}{l}
\dot{\hat{x}}=A(\theta) x+B(\theta) u+K(\theta)+L(y-\hat{y}) \\
\hat{y}=h(\hat{x}, \theta)+g(u, \theta) .
\end{array}\right.
$$

The idea is to choose $L$ independent of the temperature such that the stability properties are guaranteed for any temperature between $\theta_{\min }$ and $\theta_{\max }$. The case where $L$ depends on the temperature will be addressed in future work.

The results of Section III-A are not directly applicable to system (23) and we need to revisit Assumption 1.

Assumption 3: There exist $H_{\text {neg }}, H_{\text {pos }}, C(\theta) \in \mathbb{R}^{1 \times n_{x}}$, and $\delta_{\text {neg }}^{\max }, \delta_{\text {pos }}^{\max } \in \mathbb{R}_{\geq 0}$ such that for any $\theta \in\left[\theta_{\min }, \theta_{\max }\right]$ and for any $x, x^{\prime} \in \mathbb{R}^{n_{x}}$

$$
\begin{aligned}
& h(x, \theta)-h\left(x^{\prime}, \theta\right)=C(\theta)\left(x-x^{\prime}\right) \\
& \quad+\delta_{\text {neg }}\left(x, x^{\prime}, \theta\right) H_{\text {neg }}\left(x-x^{\prime}\right)+\delta_{\mathrm{pos}}\left(x, x^{\prime}, \theta\right) H_{\mathrm{pos}}\left(x-x^{\prime}\right),
\end{aligned}
$$

where $\quad \delta_{\text {neg }}\left(x, x^{\prime}, \theta\right) \in\left[0, \delta_{\text {neg }}^{\max }\right]$ and $\quad \delta_{\text {pos }}\left(x, x^{\prime}, \theta\right) \in$ $\left[0, \delta_{\mathrm{pos}}^{\max }\right]$.

Compared with Assumption 1, the matrix $C$ in Assumption 3 depends on $\theta$, as well as $\delta_{\text {neg }}$ and $\delta_{\text {pos }}$; however, the bounds where these two terms lie, namely, $\delta_{\text {neg }}^{\max }$ and $\delta_{\text {pos }}^{\max }$, are assumed to be independent of the temperature. This is a reasonable assumption as $\delta_{s}^{\max }$ can be chosen larger than the maximum of the upper bound of $\delta_{s}^{\max }(\theta)$ for $\theta \in\left[\theta_{\min }, \theta_{\max }\right]$. We also assume that the matrices $A(\theta)$ in (22) and (23) and $C(\theta)$ in (24) evolve in known polytopes, which is reasonable in view of the definition of the matrices $A$ in Section II and $C$ in Section III.

Assumption 4: For any $\theta \in\left[\theta_{\min }, \theta_{\max }\right]$ with $\theta_{\min }$, $\theta_{\max } \in \mathbb{R}$, there exist constant matrices $A_{1}, \ldots, A_{2^{n_{x} \times n_{x}}}$ and $C_{1}, \ldots, C_{2^{n_{x}}}$ such that $A(\theta)=\sum_{i=1}^{2^{n_{x} \times n_{x}}} \lambda_{i}(\theta) A_{i}$ and $C(\theta)=$ $\sum_{j=1}^{2^{n_{x} \times n_{x}}} \gamma_{j}(\theta) C_{j}$ with $\lambda_{i}(\theta) \in[0,1]$ for $i \in\left\{1, \ldots, 2^{n_{x} \times n_{x}}\right\}$, $\gamma_{j}(\theta) \in[0,1]$ for $j \in\left\{1, \ldots, 2^{n_{x}}\right\}, \sum_{i=1}^{2^{n_{x} \times n_{x}}} \lambda_{i}(\theta)=1$, and $\sum_{j=1}^{2^{n_{x}}} \gamma_{j}(\theta)=1$.

Using Assumption 3, the estimation error system is

$$
\dot{e}=\left(A(\theta)-L\left(C(\theta)+\delta_{\text {neg }} H_{\text {neg }}+\delta_{\text {pos }} H_{\text {pos }}\right)\right) e+E w-L z \text {. }
$$

The idea is to design a common matrix $L$ for each vertex of the polytope defined by the matrices $A_{i}$ and $C_{j}$ with $i \in\left\{1 \ldots 2^{n_{x} \times n_{x}}\right\}$ and $j \in\left\{1 \ldots 2^{n_{x}}\right\}$.

Theorem 2: Suppose Assumptions 2-4 hold, consider system (25). If there exist $\varepsilon, m_{\text {neg }}, m_{\text {pos }}, \mu_{w}, \mu_{z} \in \mathbb{R}_{>0}$, and
$P \in \mathbb{R}^{n_{x} \times n_{x}}$ symmetric and positive definite such that

$$
\left(\begin{array}{ccccc}
\mathcal{G}_{i, j}+\varepsilon I_{n_{x}} & \mathcal{K}_{\text {neg }} & \mathcal{K}_{\mathrm{pos}} & P E & -P L \\
* & -\frac{2 m_{\mathrm{neg}}}{\delta_{\mathrm{neg}}^{\max }} & 0 & 0 & 0 \\
* & * & -\frac{2 m_{\mathrm{pos}}}{\delta_{\mathrm{pos}}^{\max }} & 0 & 0 \\
* & * & * & -\mu_{w} I_{n_{w}} & 0 \\
* & * & * & * & -\mu_{z} I_{n_{z}}
\end{array}\right)<0,
$$

for all $i \in\left\{1 \ldots 2^{n_{x} \times n_{x}}\right\}$ and $j \in\left\{1 \ldots 2^{n_{x}}\right\}$, where $\mathcal{G}_{i, j}:=$ $\left(A_{i}-L C_{j}\right)^{T} P+P\left(A_{i}-L C_{j}\right), \mathcal{K}_{\text {neg }}:=-P L+m_{\text {neg }} H_{\text {neg }}^{T}$, and $\mathcal{K}_{\text {pos }}:=-P L+m_{\text {pos }} H_{\text {pos }}^{T}$, system (25) is $\mathcal{L}_{2}$-stable from $w$ and $z$ to $e$ and when $w=0$ and $z=0, e=0$ is UGES.

Sketch of Proof: The proof follows the same lines as the proof of Theorem 1 by replacing $\mathcal{G}$ by $\mathcal{G}_{i, j}$ and using $\sum_{i}^{2^{n_{x} \times n_{x}}} \lambda_{i}(\theta)=1$ and $\sum_{j}^{2^{n_{x}}} \gamma_{j}(\theta)=1$.

In practice, Theorem 2 requires to solve $2^{n_{x} \times n_{x} \times n_{x}}$ LMIs of size greater than or equal to $n_{x}+2$. However, as shown in [21, Sec. 3.3], the temperature has a negligible effect on the OCV curves (Fig. 2), which implies that $C(\theta)=C$ and the matrix $A(\theta)$ is not fully filled. This sparsity of problem formulation can be exploited to drastically reduce the size of the associated polytope.

\section{Simulation AND Discussion}

We have constructed observer (11) based on the model derived in Section II with the set of parameters given in Table I in Appendix B and assuming the temperature is constant. We have taken $N_{\text {neg }}=4$ and $N_{\text {pos }}=4$ samples with identical volumes. In this case, the system is of dimension 7; recall that one dimension is removed in observer (11) thanks to (6). This is a good compromise between model accuracy and numerical cost. The matrix $A$ is given at the bottom of the next page, $B=[0,0,1.3677,0,0,0,2.1794]$ and $K=(407.4844,0,0,0,0,0,0)$. We have constructed the matrix $L$ by solving (15) using the LMI solver Sedumi and the LMI parser Yalmip [22], considering $H_{\text {neg }}=10^{-4} \times$ $\left(\begin{array}{llllllll}0 & 0 & 0.6211 & 0 & 0 & 0 & 0\end{array}\right), H_{\mathrm{pos}}=10^{-4} \times\left(\begin{array}{lllllll}0 & 0 & 0 & 0 & 0 & 0 & 0.4184\end{array}\right)$, $\delta_{\text {neg }}^{\max }=24.5603$, and $\delta_{\mathrm{pos}}^{\max }=638.3806$, which gives $L=$ $10^{4} \times(3.1292,3.1398,3.0693,-4.9557,-4.9493,-4.9628$, $-5.0157)$. We have simulated the obtained observer (11) with an output signal not generated by models (8) and (9) but by a PDE model solved using the finite-element method, given in $[13$, Sec. 2].

The latter is more accurate than the electrochemical model described Section II (it notably does not rely on SA1), and substitutes for the real battery data. The set of parameters used to run this model is also given in Table I in Appendix B. Note that $\sigma_{e}$, the ionic conductivity, is constant and that $\sigma_{D_{e}}$, the ionic diffusion conductivity, is set to zero in order to satisfy Assumption SA2. To have a representative cycle, we have used a current profile derived from a referenced plug-in hybrid electric vehicles (PHEV) power profile given in [23] and we have repeated it seven times, as shown in Fig. 3, to test different depths of discharge. 


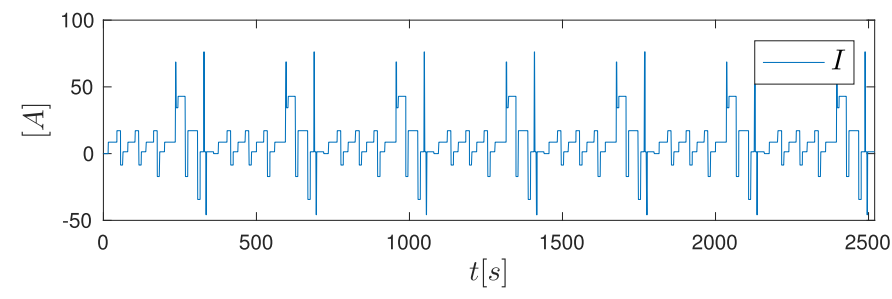

Fig. 3. PHEV current profile repeated seven times.

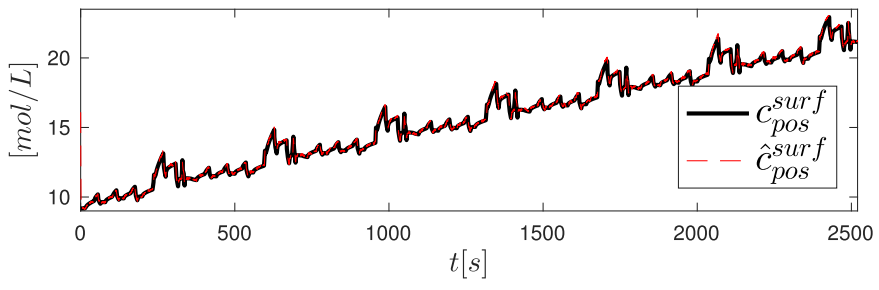

Fig. 4. Lithium surface concentration of the positive electrode.

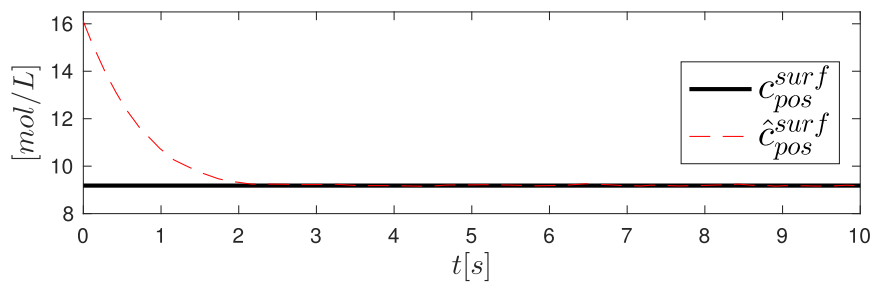

Fig. 5. Zoomed-in view of the lithium surface concentration of the positive electrode.

A white unbiased noise of $\pm 30 \mathrm{~m} V$ has been added to the output of the comprehensive PDE model to simulate the measurement noise, which is a reasonable ratio signal versus noise for embedded battery voltage measurement. The estimates $\hat{x}$ have been initialized with an error equivalent to $50 \%$ of the SOC. In the PDE model, the positive electrode is spatially homogeneous, hence, only one surface concentration is given as reference in Figs. 4 and 5. Fig. 5 shows that the initial error takes about $2 s$ to vanish. The negative electrode in the PDE model is not spatially homogeneous, hence, the lithium concentration near the separator and near the collector differs. Both the surface lithium concentration at the collector junction and at the separator junction of the negative electrode are shown in Figs. 6 and 7. Fig. 7 is a zoomed-in view of Fig. 6 in which we see that the estimated lithium surface concentration at the negative electrode evolves in a domain bounded by the surface concentrations at the sides of the electrode and is often more representative of what happens near the collector.

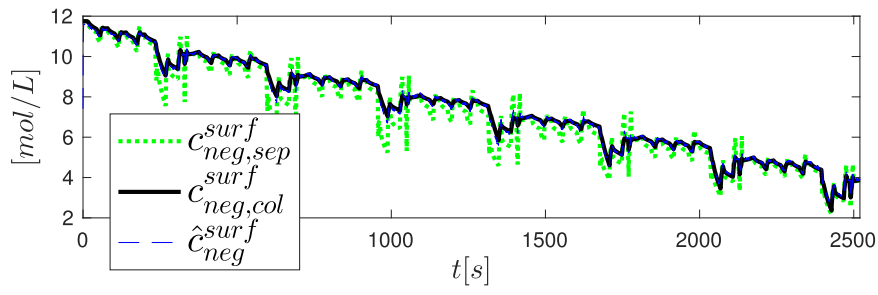

Fig. 6. Lithium surface concentration of the nonhomogeneous negative electrode

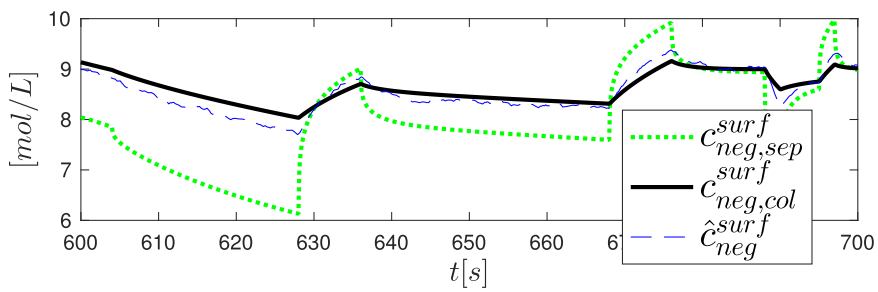

Fig. 7. Zoomed-in view of the lithium surface concentration of the nonhomogeneous negative electrode.

Estimating these surface concentrations is one of the main advantages of this electrochemical model-based approach as these are responsible of the OCV value, see (9), and play a key role in the BMS. The simulation results given in Figs. 4 and 6 clearly show the fastness and efficiency of the proposed observer as well as its robustness to noisy measurements. We can then exploit the state estimates provided by the observer to estimate the SOC. When the states of the model (10) are estimated, the SOC can be estimated,

$$
\widehat{\operatorname{SOC}}(t)=100 \times \frac{\bar{c}^{\mathrm{pos}}(t)-c_{0}^{\mathrm{pos}}}{c_{100}^{\mathrm{pos}}-c_{0}^{\mathrm{pos}}},
$$

with $\bar{c}^{\mathrm{pos}}(t)=\sum_{i=1}^{N_{\mathrm{pos}}} \hat{c}_{i}^{\mathrm{pos}}(t) V_{i}^{\mathrm{pos}} / V_{\mathrm{total}}^{\mathrm{pos}}$, where $\hat{c}_{i}^{\mathrm{pos}}$ stands for the components of $\hat{x}$, corresponding to lithium concentrations in the positive electrode. We recall that $c_{0}^{s}$ and $c_{100}^{s}$ stand for lithium concentration at SOC $=0 \%$ and $\mathrm{SOC}=100 \%$, respectively, and that numerical values of these are given in Table I in Appendix B.

To challenge this estimated SOC, we have used the coulomb metric SOC as reference, which consists in integrating the current applied to the battery over the time,

$$
\begin{aligned}
\operatorname{SOC}(t) & =\operatorname{SOC}(0) \\
& -\frac{100}{F\left(c_{0}^{\mathrm{pos}}-c_{100}^{\mathrm{pos}}\right) A_{\mathrm{cell}} d_{\mathrm{pos}} \varepsilon_{\mathrm{pos}}} \times \int_{0}^{t} i_{\text {cell }}(\tau) d \tau .
\end{aligned}
$$

While (28) is often used in practice, its weakness is the accumulation of inaccuracy due to the integrator. This weakness

$$
A=10^{-2} \times\left(\begin{array}{ccccccc}
-2.48 & 0.73 & -0.58 & -0.37 & -0.37 & -0.37 & -0.37 \\
1.32 & -3.48 & 2.17 & 0 & 0 & 0 & 0 \\
0 & 2.17 & -2.17 & 0 & 0 & 0 & 0 \\
0 & 0 & 0 & -1.08 & 1.08 & 0 & 0 \\
0 & 0 & 0 & 1.08 & -3.51 & 2.44 & 0 \\
0 & 0 & 0 & 0 & 2.44 & -6.44 & 4.01 \\
0 & 0 & 0 & 0 & 0 & 4.01 & -4.01
\end{array}\right)
$$




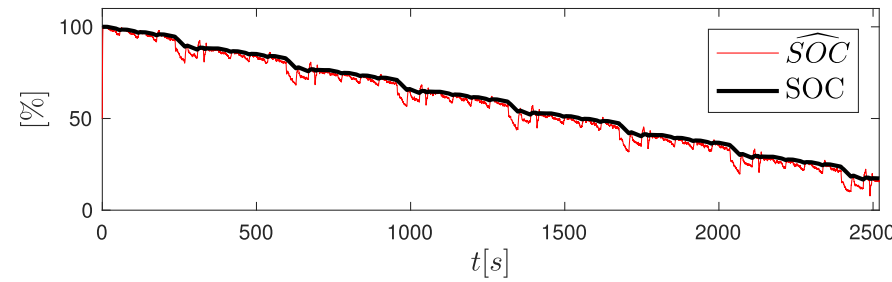

Fig. 8. SOC estimation on a normalized PHEV current profile for a fully charged battery with $50 \%$ of initial SOC error and under measurement noise.

TABLE I

Physical Parameters of the Electrochemical Model From [5]

\begin{tabular}{|c|c|c|}
\hline Parameter [units] & $\begin{array}{l}\text { Negative } \\
\text { electrode }\end{array}$ & $\begin{array}{l}\text { Positive } \\
\text { electrode }\end{array}$ \\
\hline Solid phase volume fraction $\varepsilon[-]$ & 0.332 & 0.330 \\
\hline Cell area $\mathcal{A}_{\text {cell }}\left[\mathrm{m}^{2}\right]$ & 1.0452 & 1.0452 \\
\hline $\begin{array}{l}\text { Max. solid phase concentration } c_{s}^{\max } \\
{\left[\mathrm{mol} . \mathrm{L}^{-1}\right]}\end{array}$ & 16.1 & 23.9 \\
\hline Thickness $d[\mu m]$ & 50 & 36 \\
\hline Lithium diffusion coef $D\left[\mathrm{~m}^{2} . \mathrm{s}^{-1}\right]$ & $2 \times 10^{-16}$ & $3.6 \times 10^{-16}$ \\
\hline Electrolytic diffusion coef $D_{e}\left[\mathrm{~m}^{2} \cdot \mathrm{s}^{-1}\right]$ & $2.6 \times 10^{-10}$ & $2.6 \times 10^{-10}$ \\
\hline Exchange current density $j_{0}\left[A . m^{-2}\right]$ & 36 & 26 \\
\hline Number of sample $N$ [-] & 4 & 4 \\
\hline Particle radius $R[\mu \mathrm{m}]$ & 1 & 1 \\
\hline Electronic conductivity $\sigma\left[S . m^{-1}\right]$ & 100 & 10 \\
\hline Ionic conductivity $\sigma_{e}\left[S . m^{-1}\right]$ & 0.6329 & 0.6329 \\
\hline Ionic diffusion conductivity $\sigma_{D_{e}}\left[S . m^{-1}\right]$ & 0 & 0 \\
\hline Additional resistivity $\Omega_{a d d}[\Omega]$ & 0 & 0 \\
\hline $\begin{array}{l}\text { Lithium concentration at } S O C=0 \% c_{0} \\
{\left[m o l . L^{-1}\right]}\end{array}$ & 3.069 & 23.01 \\
\hline $\begin{array}{l}\text { Lithium concentration at } S O C=100 \% \\
c_{100}\left[\mathrm{~mol}^{-1} \mathrm{~L}^{-1}\right]\end{array}$ & 11.75 & 9.182 \\
\hline
\end{tabular}

does not impact this simulation as the current is perfectly known in our simulations.

Fig. 8 shows that under noise and significant initial error, the estimated SOC in (27) fastly and robustly converges to a small neighborhood of the true one.

\section{CONCLUSION}

We have presented a nonlinear observer for a finite dimensional SPM model of a battery lithium ion, whose design relies on circle-criterion-like arguments. The global stability and robustness of the nonlinear observer are ensured, provided that an LMI holds, which can be used to design the observation gain. Simulation results on a comprehensive PDE model of the battery show that the obtained estimates are accurate even in the presence of noise measurements. Several applications can then come out as SOC, as illustrated in simulations in Section IV, SOH, or smarter safety and aging limitations.

\section{APPENDiX A \\ Detailed OutPut of The System}

For any $u \in \mathbb{R}$, the current-dependent component $g(u)$ of the electrochemical model output mentioned in (9) is $g(u):=g_{1}(u)+g_{2}(u)+g_{3}(u)$. The term $g_{1}$ represents the activation overpotential of the positive electrode, $g_{2}$ is the activation overpotential of the negative electrode, and $g_{3}$ are the electronic resistivity terms of the battery; they are
TABLE II

SYMBOL DESCRIPTION

\begin{tabular}{|c|c|}
\hline \multicolumn{2}{|c|}{ Latin letters } \\
\hline$\varepsilon$ & Volume fraction $[-]$ \\
\hline$\sigma$ & Conductivity $\left[S . m^{-1}\right]$ \\
\hline $\mathcal{A}$ & Active surface $\left[\mathrm{m}^{2}\right]$ \\
\hline$a$ & Active surface per volume unit $\left[\mathrm{m}^{-1}\right]$ \\
\hline$c$ & Lithium concentration $\left[\mathrm{mol} . \mathrm{m}^{-3}\right]$ \\
\hline$D$ & Lithium Diffusion coefficient $\left[m^{2} \cdot s^{-1}\right]$ \\
\hline$F$ & Faraday's constant $\left[C \cdot \mathrm{mol}^{-1}\right]$ \\
\hline$N$ & Order of the model $[-]$ \\
\hline$Q$ & Thermodynamic capacity $[A . h]$ \\
\hline$R$ & Radius $[m]$ \\
\hline$r$ & Radial coordinate $[\mathrm{m}]$ \\
\hline $\mathcal{R}$ & Gaz constant $\left[J \cdot K^{-1} \cdot \mathrm{mol}^{-1}\right]$ \\
\hline$S$ & Surface $\left[m^{2}\right]$ \\
\hline$t$ & Time $[s]^{\circ}$ \\
\hline \multicolumn{2}{|c|}{ Subscript and superscript } \\
\hline cell & Related to battery cell \\
\hline $\mathrm{col}$ & Collector side \\
\hline neg & Related to negative electrode \\
\hline$n_{x}$ & Size of vector $x$ \\
\hline pos & Related to positive electrode \\
\hline$s$ & Solid phase, stands for neg or pos \\
\hline sep & Separator side \\
\hline surf & Surface of an electrode \\
\hline total & the whole entity \\
\hline 0 & $0 \%$ of capacity left \\
\hline 100 & $100 \%$ of capacity left \\
\hline
\end{tabular}

defined as

$$
\begin{aligned}
& g_{1}(u)=-2 \frac{\mathcal{R} T}{F} \operatorname{Argsh}\left(\frac{R_{\mathrm{pos}}}{6 \varepsilon_{\mathrm{pos} j}^{\mathrm{pos}} \mathcal{A}_{\text {cell }} d_{\mathrm{pos}}} u\right) \\
& g_{2}(u)=2 \frac{\mathcal{R} T}{F} \operatorname{Argsh}\left(\frac{R_{\mathrm{neg}}}{6 \varepsilon_{\mathrm{neg}} j_{0}^{\text {neg }} \mathcal{A}_{\text {cell }} d_{\text {neg }}} u\right) \\
& g_{3}(u)=-\left(\frac{1}{2 \mathcal{A}_{\text {cell }}}\left(\frac{d_{\text {neg }}}{\sigma_{\text {neg }}}+\frac{d_{\mathrm{pos}}}{\sigma_{\mathrm{pos}}}\right)+\Omega_{\text {add }}\right) u
\end{aligned}
$$

with $\operatorname{Argsh}(\xi)=\ln \left(\xi+\sqrt{\xi^{2}+1}\right)$ for $\xi \in \mathbb{R}$.

\section{APPENDIX B}

See Tables I and II.

\section{REFERENCES}

[1] B. Saha, K. Goebel, S. Poll, and J. Christophersen, "Prognostics methods for battery health monitoring using a Bayesian framework," IEEE Trans. Instrum. Meas., vol. 58, no. 2, pp. 291-296, Feb. 2009.

[2] G. L. Plett, "Extended Kalman filtering for battery management systems of LiPB-based HEV battery packs: Part 3. State and parameter estimation," J. Power Sour., vol. 134, no. 2, pp. 277-292, 2004.

[3] M. Urbain, S. Rael, B. Davat, and P. Desprez, "State estimation of a lithium-ion battery through Kalman filter," in Proc. Power Electron. Specialists Conf., Jun. 2007, pp. 2804-2810.

[4] M. Doyle, T. F. Fuller, and J. Newman, "Modeling of galvanostatic charge and discharge of the lithium/polymer/insertion cell," J. Electrochem. Soc., vol. 140, no. 6, pp. 1526-1533, 1993.

[5] K. Smith and C.-Y. Wang, "Power and thermal characterization of a lithium-ion battery pack for hybrid-electric vehicles," J. Power Sour., vol. 160, no. 1, pp. 662-673, 2006.

[6] E. Prada, D. Di Domenico, Y. Creff, J. Bernard, V. Sauvant-Moynot, and F. Huet, "A simplified electrochemical and thermal aging model of $\mathrm{LiFePO}_{4}$-graphite Li-ion batteries: Power and capacity fade simulations," J. Electrochem. Soc., vol. 160, no. 4, pp. A616-A628, 2013.

[7] C. Zou, C. Manzie, and D. Nešić, "A framework for simplification of PDE-based lithium-ion battery models," IEEE Trans. Control Syst. Technol., vol. 24, no. 5, pp. 1594-1609, Sep. 2016. 
[8] D. Di Domenico, A. Stephanopoulou, and G. Fiengo, "Lithium-ion battery state of charge and critical surface charge estimation using an electrochemical model-based extended Kalman filter," J. Dyn. Syst. Meas. Control, vol. 132, no. 6, p. 061302, 2010.

[9] S. J. Moura, F. B. Argomedo, R. Klein, A. Mirtabatabaei, and M. Krstic, "Battery state estimation for a single particle model with electrolyte dynamics," IEEE Trans. Control Syst. Technol., vol. 25, no. 2, pp. 453-468, Mar. 2017.

[10] S. J. Moura, N. A. Chaturvedi, and M. Krstić, "PDE estimation techniques for advanced battery management systems-Part II: SOH identification," in Proc. Amer. Control Conf. (ACC), 2012, pp. 566-571.

[11] S. Santhanagopalan and R. E. White, "State of charge estimation using an unscented filter for high power lithium ion cells," Int. J. Energy Res., vol. 34, no. 2, pp. 152-163, 2009.

[12] S. Dey, B. Ayalew, and P. Pisu, "Nonlinear robust observers for state-of-charge estimation of lithium-ion cells based on a reduced electrochemical model," IEEE Trans. Control Syst. Technol., vol. 23, no. 5, pp. 1935-1942, Sep. 2015.

[13] S. Raël and M. Hinaje, "Using electrical analogy to describe mass and charge transport in lithium-ion batteries," J. Power Sour., vol. 222, pp. 112-122, Jan. 2013

[14] X. Fan and M. Arcak, "Observer design for systems with multivariable monotone nonlinearities," Syst. Control Lett., vol. 50, no. 4, pp. 319-330, 2003.
[15] A. Johansson and A. Medvedev, "An observer for systems with nonlinear output map," Automatica, vol. 39, no. 5, pp. 909-918, 2003.

[16] M. Arcak and P. Kokotović, "Nonlinear observers: A circle criterion design and robustness analysis," Automatica, vol. 37, no. 12, pp. 1923-1930, 2001

[17] M. Chong, R. Postoyan, D. Nešić, L. Kuhlmann, and A. Varsavsky, "A robust circle criterion observer with application to neural mass models," Automatica, vol. 48, no. 11, pp. 2986-2989, 2012.

[18] P. G. Blondel, R. Postoyan, S. Raël, S. Benjamin, and P. Desprez, "Observer design for an electrochemical model of lithium ion batteries based on a polytopic approach," IFAC-PapersOnLine, vol. 50, no. 1, pp. 8127-8132, Jul. 2017.

[19] M. Arcak and P. Kokotovic, "Observer-based control of systems with slope-restricted nonlinearities," IEEE Trans. Autom. Control, vol. 46, no. 7, pp. 1146-1150, Jul. 2001.

[20] H. Khalil, Nonlinear Systems, 3rd ed. Englewood Cliffs, NJ, USA: Prentice-Hall, 2002.

[21] A. Samba, "Battery electrical vehicles-analysis of thermal modelling and thermal management," Ph.D. dissertation, Normandie Univ., Caen, France, 2015.

[22] J. Lofberg, "YALMIP: A toolbox for modeling and optimization in MATLAB," in Proc. IEEE Int. Symp. Comput. Aided Control Syst. Design, Sep. 2004, pp. 284-289.

[23] J. R. Belt, "Battery test manual for plug-in hybrid electric vehicles," Idaho Nat. Lab., Idaho Falls, ID, USA, Tech. Rep. INL/EXT-07-12536REV1, 2010. 